\title{
Tyrosine Kinase Inhibitors Imatinib and Erlotinib Increase Apoptosis of Antimitotic Drug-resistant KBV20C Cells Without Inhibiting P-gp
}

\author{
JI YEONG KIM, HYUNG SIK KIM and SUNGPIL YOON
}

\author{
School of Pharmacy, Sungkyunkwan University, Suwon, Republic of Korea
}

\begin{abstract}
Background/Aim: This study investigated drugs able to sensitize P-glycoprotein (P-gp)-overexpressing resistant KBV20C cancer cells to vincristine or eribulin treatment and assessed their associated mechanisms of action. Materials and Methods: Eight tyrosine kinase inhibitors (lapatinib, gefitinib, imatinib, erlotinib, nilotinib, pazopanib, cediranib, and vandetanib) and one serine/threonine kinase inhibitor (selumetinib) were evaluated for their sensitizing effects on vincristine-resistant $K B V 20 C$ cells at relatively low doses. Fluorescence-activated cell sorting, annexin $V$ analyses, and rhodamine uptake tests were further performed to investigate their mechanisms of action. Results: Cotreatment of KBV20C cells with lapatinib, gefitinib, imatinib, or erlotinib at low doses highly sensitized them to vincristine treatment. These drugs reduced cellular viability, increased $G_{2}$ arrest, and up-regulated apoptosis when co-administered with vincristine. In a detailed quantitative analysis using lower doses, we demonstrated that lapatinib, with high P-gp inhibitory activity, yielded the best pairing for sensitizing $P$-gp-overexpressing KBV20C cells to vincristine. Cotreatment with eribulin and lapatinib, gefitinib, or erlotinib also increased the sensitivity of KBV20C cells, suggesting that they can be combined with other antimitotic drugs to sensitize resistant cancer cells. Lapatinib was shown to have a higher $P$-gp-inhibitory activity than verapamil, even at lower doses, indicating that its sensitizing of cells to vincristine involves its $P$-gp-inhibitory effects. However, interestingly, imatinib-and erlotinib-sensitizing of cells to vincristine appears to be independent of their P-gp inhibition. Conclusion: These findings provide valuable information regarding the sensitizing
\end{abstract}

Correspondence to: Sungpil Yoon, Ph.D., School of Pharmacy, Sungkyunkwan University, 2066 Seobu-ro, Jangan-gu, Suwon, Gyeonggi-do, 16419, Republic of Korea. Tel: +82 1055024893, Fax: +82 312928800, e-mail: syoon88@gmail.com

Key Words: Lapatinib, gefitinib, erlotinib, tyrosine kinase inhibitors, cancer, P-gp, drug resistance. of drug-resistant cells and indicate that imatinib and erlotinib may be used in patients with potentially resistant cancer without any toxic effects from P-gp inhibition.

Antimitotic drugs inhibit mitosis by targeting microtubules and preventing their polymerization or depolymerization. Paclitaxel, docetaxel, vincristine vinorelbine, vinblastine, and eribulin are examples of antimitotic drugs (1-4). Although antimitotic drugs are widely used to treat cancer, cancer cells can develop resistance to these drugs in various ways. P-glycoprotein (P-gp) overexpression is a well-known mechanism of resistance to antimitotic drugs. P-gp is a membrane channel that can pump out antimitotic drugs, thus enabling cells to avoid drug-induced toxicity (5-8). Identifying mechanisms to sensitize cancer cells that overexpress $\mathrm{P}$-gp can lead to better treatment in patients who develop resistance to antimitotic drugs. Although P-gp inhibitors have been developed, their toxicity in normal cells leads to treatment failure. Therefore, it is important to investigate novel therapeutic options without $\mathrm{P}$-gp inhibition for P-gp-overexpressing drug-resistant cancer cells.

Thus, the urgent need for pharmacological treatments for P-gp-overexpressing resistant cancer can be effectively addressed if novel mechanisms of approved anti-cancer drugs are identified because these drugs can be used without further toxicity evaluation (9-11).

Tyrosine kinase inhibitors (TKIs) generally target the epidermal growth factor receptor (EGFR) family and have been developed as a cancer therapy for preventing growth factor signaling in various cancer models (12-14). They are reversible competitors of ATP for binding at the intracellular catalytic domain of the EGFRs. In addition, TKIs act as inhibitors of P-gp in cancer cells $(15,16)$. These drugs have also been reported to sensitize drug-resistant cancer cells (17, 18). However, the exact mechanisms of action of individual TKIs have not yet been investigated.

Based on a literature search, we identified eight TKIs (lapatinib, gefitinib, imatinib, erlotinib, nilotinib, pazopanib, cediranib, and vandetanib) and one serine/threonine kinase 
inhibitor (selumetinib) $(12-14,19)$ which might have sensitizing effects on $\mathrm{P}$-gp-overexpressing drug-resistant KBV20C cancer cells. We also investigated the mechanisms involved in the sensitizing of resistant cancer cells. As these agents are already in clinical use as targeting anticancer drugs, these results can contribute to the development of therapies using co-treatment with TKIs for highly drugresistant tumors.

\section{Materials and Methods}

Reagents and cell culture. Rhodamine123 (Rhodamine) and verapamil were purchased from Sigma-Aldrich (St. Louis, MO, USA). Vincristine was purchased from Enzo Life Sciences (Farmingdale, NY, USA). Lapatinib, gefitinib, imatinib, erlotinib, nilotinib, pazopanib, cediranib, vandetanib, and selumetinib were purchased from Selleckchem (Houston, TX, USA). Aqueous solutions of eribulin (Eisai Korea, Seoul, South Korea) were obtained from the National Cancer Center in South Korea.

Human oral squamous carcinoma cell line, KB, and its multidrug-resistant subline, KBV20C, were obtained from Dr. Yong Kee Kim (College of Pharmacy, Sookmyung Women's University, Seoul, South Korea) and have been described elsewhere (20-25). All cell lines were cultured in RPMI 1640 containing 10\% fetal bovine serum, $100 \mathrm{U} / \mathrm{ml}$ penicillin, and $100 \mu \mathrm{g} / \mathrm{ml}$ streptomycin (WelGENE, Daegu, South Korea).

Microscopic observation. Cells were grown to $40 \%-50 \%$ confluence in $60-\mathrm{mm}$ diameter dishes and treated with $5 \mu \mathrm{M}$ lapatinib, gefitinib, imatinib, erlotinib, nilotinib or pazopanib, or $10 \mu \mathrm{M}$ verapamil alone and in combination with $50 \mathrm{ng} / \mathrm{ml}(60 \mathrm{nM})$ eribulin or $5 \mathrm{nM}$ vincristine for $24 \mathrm{~h}$. The medium was removed, and phosphate-buffered saline (PBS) was added into each dish. Attached cells were examined immediately in two independent experiments using an ECLIPSE Ts2 inverted routine microscope (Nikon, Tokyo, Japan) with a $4 \times$ or a $10 \times$ objective lens (Nikon's Microscopy U).

Rhodamine uptake tests. The tests used to assess the ability of a drug to inhibit P-gp were based on a previously described method (20-25). Briefly, cells were grown to $40-50 \%$ confluence in $60-\mathrm{mm}$ diameter dishes and treated with $5 \mu \mathrm{M}$ lapatinib, gefitinib, imatinib, erlotinib, nilotinib, pazopanib, cediranib, vandetanib or selumetinib, or $10 \mu \mathrm{M}$ verapamil for $4 \mathrm{~h}$ or $24 \mathrm{~h}$ at $37^{\circ} \mathrm{C}$. Cells were then incubated with $2 \mu \mathrm{g} / \mathrm{ml}$ rhodamine for $1 \mathrm{~h} 30 \mathrm{~min}$ at $37^{\circ} \mathrm{C}$. The medium was removed, and the cells were washed with PBS. The stained cells were analyzed in two independent experiments using a Guava EasyCyte Plus Flow Cytometer (Merck Millipore, Burlington, MA, USA).

Fluorescence-activated cell sorting (FACS) analysis. FACS analysis was performed as previously described (20-25). Cells were grown to $40 \%-50 \%$ confluence in $60-\mathrm{mm}$ diameter dishes and treated $5 \mu \mathrm{M}$ lapatinib, gefitinib or erlotinib, or $10 \mu \mathrm{M}$ verapamil alone and in combination with $5 \mathrm{nM}$ vincristine for $24 \mathrm{~h}$. The cells were then dislodged by trypsin and pelleted by centrifugation. The pelleted cells were washed thoroughly with PBS, suspended in $75 \%$ ethanol for at least $1 \mathrm{~h}$ at $4^{\circ} \mathrm{C}$, washed with PBS, and re-suspended in a cold propidium iodide (PI) staining solution $(100 \mu \mathrm{g} / \mathrm{ml}$ RNase A and 50 $\mu \mathrm{g} / \mathrm{ml} \mathrm{PI}$ in PBS) for $30 \mathrm{~min}$ at $37^{\circ} \mathrm{C}$. The stained cells were analyzed in two independent experiments for relative DNA content using a Guava EasyCyte Plus Flow Cytometer (Merck Millipore).

Annexin $V$ analysis. Annexin $\mathrm{V}$ analysis was conducted using annexin V-fluorescein isothiocyanate (FITC) staining kit (BD Bioscience, Franklin, NJ, USA) as previously described (20-25). Cells were grown to $40-50 \%$ confluence in $60-\mathrm{mm}$ diameter dishes and treated with $5 \mu \mathrm{M}$ lapatinib, gefitinib or erlotinib, or $10 \mu \mathrm{M}$ verapamil alone and in combination with $5 \mathrm{nM}$ vincristine for $24 \mathrm{~h}$. The cells were then dislodged by trypsin and pelleted by centrifugation. The pelleted cells were washed with PBS. Cells in $100 \mu \mathrm{l}$ of binding buffer received $5 \mu \mathrm{l}$ of Annexin V-FITC and $5 \mu \mathrm{l}$ of PI and were then incubated for $15 \mathrm{~min}$ at room temperature. The stained cells were analyzed in two independent experiments using a Guava EasyCyte Plus Flow Cytometer (Merck Millipore).

Cell-viability assay. Cell proliferation was measured by a colorimetric assay using the EZ-CyTox cell viability assay kit (Daeillab, South Korea) according to the manufacturer's instructions. Briefly, cells were grown to $30-40 \%$ confluence in wells of 96-well plates and treated with $5 \mu \mathrm{M}$ of lapatinib, gefitinib, imatinib, erlotinib, nilotinib, pazopanib, cediranib, vandetanib or selumetinib, or $10 \mu \mathrm{M}$ verapamil alone and in combination with $5 \mathrm{nM}$ vincristine for $48 \mathrm{~h}$. They were then incubated with $10 \mu \mathrm{l}$ of EZ-CyTox solution for $1-2 \mathrm{~h}$ at $37^{\circ} \mathrm{C}$. Absorbance at $450 \mathrm{~nm}$ was determined immediately using VERSA MAX Microplate Reader (Molecular Devices Corp., Sunnyvale, CA, USA). All experiments were performed at least in triplicate and repeated twice.

Statistical analysis. Data are presented as mean \pm standard deviation (S.D.). Statistical analysis was performed using Student's $t$-test and one-way analysis of variance (ANOVA) followed by a multiplecomparison test. Results were considered statistically significant compared to those of the control when $p<0.05$.

\section{Results}

Lapatinib, gefitinib, imatinib, and erlotinib sensitize resistant KBV20C cancer cells to vincristine treatment better than other TKIs. We planned to identify specific TKIs for sensitizing resistant cancer cells at relatively low doses. Previously it was shown that TKIs enhanced the sensitivity of P-gp-overexpressing resistant cancer (15-18). We considered TKIs that are already in clinical use because they can be readily used without the need for further toxicity studies after elucidation of their mechanism of action on resistant cancer cells. Therefore, we performed further detailed analysis with eight TKIs: Lapatinib, gefitinib, imatinib, erlotinib, nilotinib, pazopanib, cediranib, and vandetanib. In addition, we tested one serine/threonine kinase inhibitor, selumetinib (19). We focused on TKIs that sensitize P-gp-overexpressing resistant cancer cells at low doses. We evaluated the TKIs in combination with vincristine, an antimitotic drug that is routinely used as a chemotherapeutic agent in cancer $(1,2)$, using KBV20C resistant cancer cells, which present a vincristine-resistant phenotype due to P-gp overexpression (26-28). 

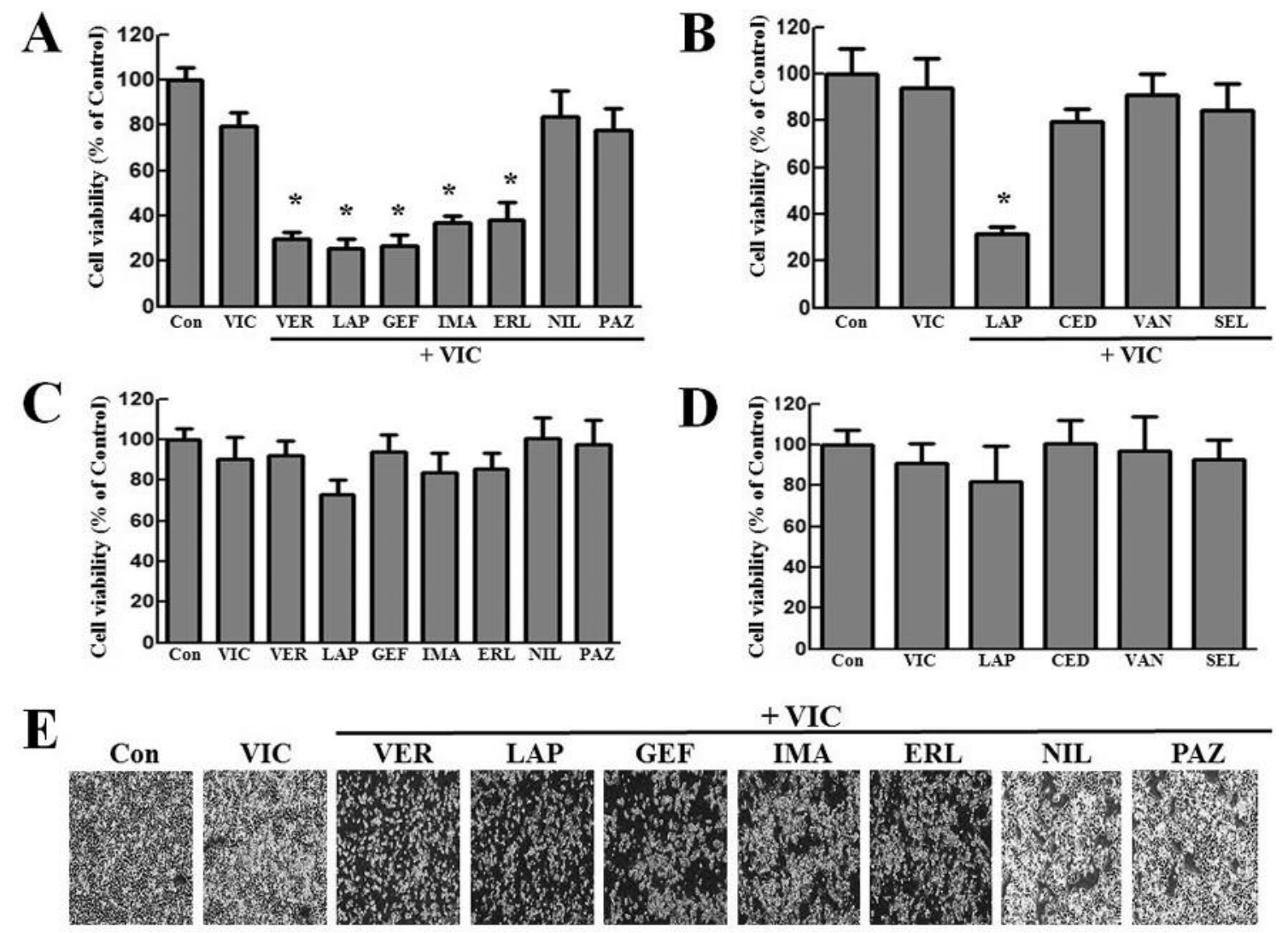

Figure 1. Lapatinib (LAP), gefitinib (GEF), imatinib (IMA), erlotinib (ERL) sensitize vincristine (VIC)-treated resistant KBV20C cancer cells better than other TKIs. A-D: KBV20C cells were plated on 96-well plates and grown to 30-40\% confluence. The cells were then stimulated for $48 \mathrm{~h}$ with $10 \mu M$ verapamil, or $5 \mu$ M lapatinib, gefitinib, imatinib, erlotinib, nilotinib (NIL), pazopanib (PAZ), cediranib (CED), vandetanib (VAN), or selumetinib (SEL) alone and in combination with $5 \mathrm{nM}$ vincristine, or with $0.1 \%$ dimethyl sulfoxide (Con). Cell viability assay was performed as described in the Materials and Methods. The data are presented as the mean $\pm S . D$. of at least two experiments repeated in triplicate experiments. Statistical analysis was conducted using one-way analysis of variance (ANOVA) followed by multiple-comparison test; *Significantly different at $p<0.05$ compared to the corresponding control. E: KBV20C cells were grown on 60 mm-diameter dishes and treated as described above. After 1 day, cells were examined using an inverted microscope at $\times 4$ magnification (scale bar $=100 \mu \mathrm{m}$ ).

Firstly, we tested whether co-treatment with TKIs increased sensitivity of KBV20C cells to vincristine treatment using a quantitative cell viability test. As seen in Figure 1A and B, lapatinib, gefitinib, imatinib, and erlotinib highly reduced viability of vincristine-treated resistant KBV20C cells. The viability of cells treated with the vincristine with lapatinib, gefitinib, imatinib, and erlotinib combinations was $>60 \%$ as compared to that of the control. We compared the effects of TKIs with those of the well-known P-gp inhibitor verapamil. Lapatinib $(5 \mu \mathrm{M})$, gefitinib $(5 \mu \mathrm{M})$, and verapamil $(10 \mu \mathrm{M})$ were similarly effective when applied with vincristine in reducing KBV20C cell viability (Figure 1A and B). There was no difference between the control and monotherapy with TKIs (Figure 1C and D), suggesting that there were synergistic effects of vincristine in combination with lapatinib, gefitinib, imatinib, and erlotinib in the VIC-resistant cancer cells. We confirmed the results of the viability tests by microscopic observation. As shown in Figure 1E, when combined with vincristine, $5 \mu \mathrm{M}$ of lapatinib, gefitinib, imatinib, or erlotinib showed sensitizing effects similar to those of verapamil.

Altogether, when we analyzed eight known TKIs to identify those sensitizing P-gp-overexpressing resistant cancer cells, we observed that lapatinib, gefitinib, imatinib, and erlotinib at low doses elicited highly sensitizing effects with greater reduction of viability than the other TKIs. We conclude that low doses of lapatinib, gefitinib, imatinib, or erlotinib can be used to reduce drug toxicity and sensitize resistant cancer cells to vincristine.

Co-treatment with vincristine and TKIs sensitizes resistant KBV20C cells via apoptosis through induction of $G_{2}$ arrest. To further clarify the mechanism of action of co-treatment 


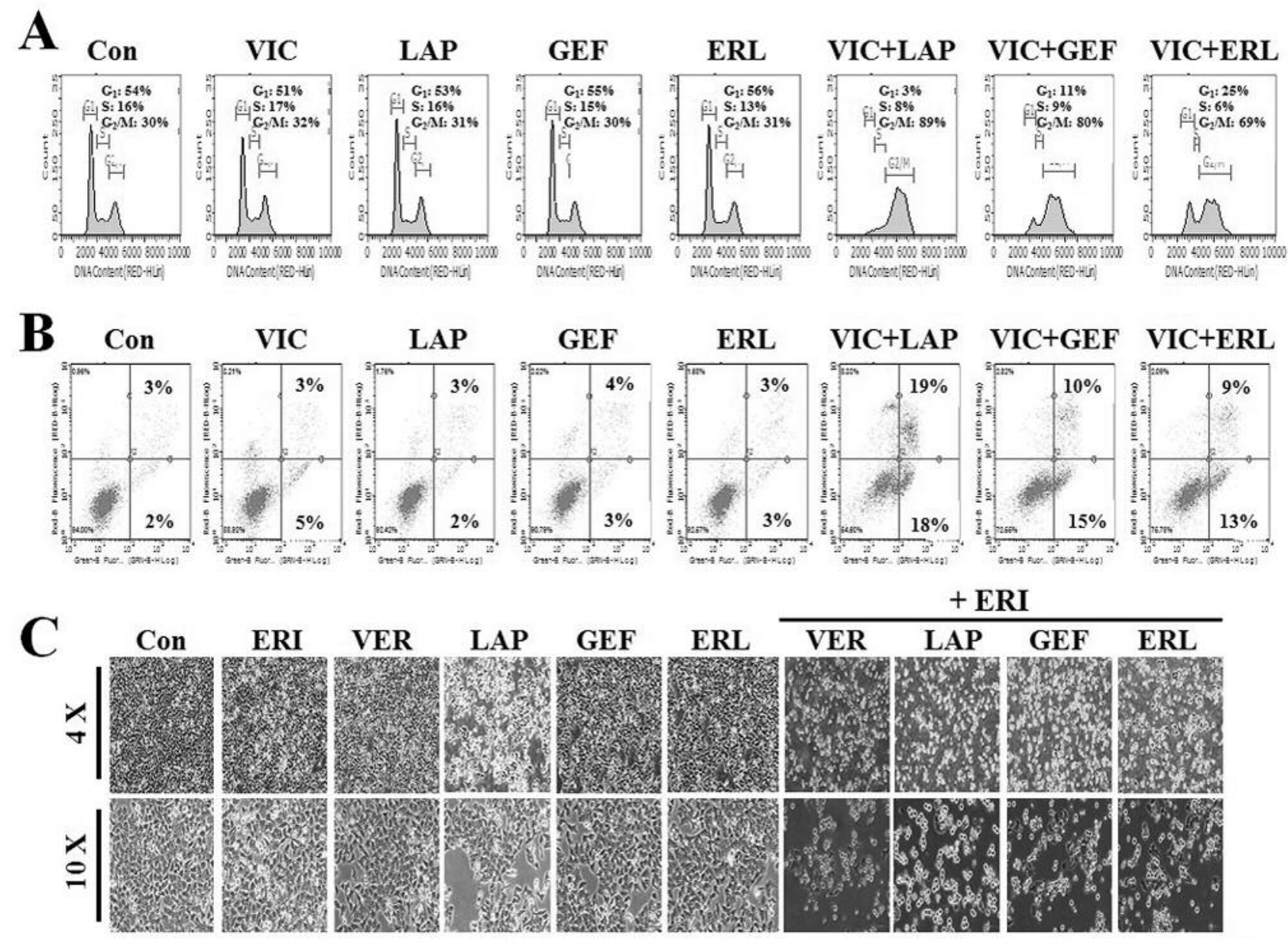

Figure 2. Vincristine (VIC) with tyrosine kinase inhibitors sensitize resistant KBV20C cells via apoptosis through induction of $G_{2}$ arrest. A: KBV20C cells were grown on $60 \mathrm{~mm}$-diameter dishes and treated with $5 \mu \mathrm{M}$ lapatinib (LAP), $5 \mu \mathrm{M}$ gefitinib (GEF), or $2.5 \mu M$ erlotinib (ERL) alone and in combination with $5 \mathrm{nM}$ vincristine, or $0.1 \%$ dimethyl sulfoxide (DMSO; Con). After 24 h, fluorescence-activated cell sorting analyses were performed as described in the Materials and Methods. B: KBV20C cells were grown on $60 \mathrm{~mm}$-diameter dishes and stimulated with $5 \mu M$ lapatinib, gefitinib or erlotinib alone and in combination with $5 \mathrm{nM}$ vincristine, or with $0.1 \%$ DMSO (Con). After 24 h, annexin V analyses were performed as described in the Materials and Methods. C: KBV20C cells were grown on $60 \mathrm{~mm}$-diameter dishes and treated with $10 \mu M$ verapamil, or $5 \mu M$ lapatinib, gefitinib, or erlotinib alone and in combination with $50 \mathrm{ng} / \mathrm{ml}$ eribulin, or with $0.1 \%$ DMSO (Con). After $24 \mathrm{~h}$, cells were examined using an inverted microscope at $\times 4$ or $x 10$ magnification (scale bar $=100 \mu \mathrm{m}$ ).

with vincristine and TKIs, we performed FACS analyses. As shown in Figure 2A, co-treatments using vincristine with lapatinib, gefitinib, and erlotinib considerably increased the number of cells in $\mathrm{G}_{2}$ arrest compared to that observed after monotherapy with either agent. This indicates that cell-cycle arrest resulted in the reduction of cellular viability.

Using annexin $\mathrm{V}$ analysis, we also tested whether combinations increased cell death by apoptosis. As seen in Figure 2B, apoptotic cell death greatly increased after cotreatment with vincristine with lapatinib, gefitinib, and erlotinib. This indicates that reduced $\mathrm{G}_{2}$ arrest contributed to increased apoptotic death. Annexin $\mathrm{V}$ staining was also analyzed in detail. As seen in Figure 2B, the proportion of apoptotic cells (in both early and late phases) after vincristine treatment with lapatinib was about $37 \%, 25 \%$ when combined with gefitinib, and $22 \%$ with erlotinib, suggesting that lapatinib sensitized cells to vincristine much better than did gefitinib and erlotinib. Overall, we demonstrated that co-treatments with lapatinib, gefitinib, and erlotinib sensitized vincristine-treated resistant KBV20C cells via $\mathrm{G}_{2}$ cell-cycle arrest and apoptosis.

TKIs increase sensitivity of KBV2OC cells to eribulin when used at low doses. We also investigated whether lapatinib, gefitinib, or erlotinib could be used in combination with other antimitotic drugs. We tested eribulin, an antimitotic drug which has been recently developed and used in the 

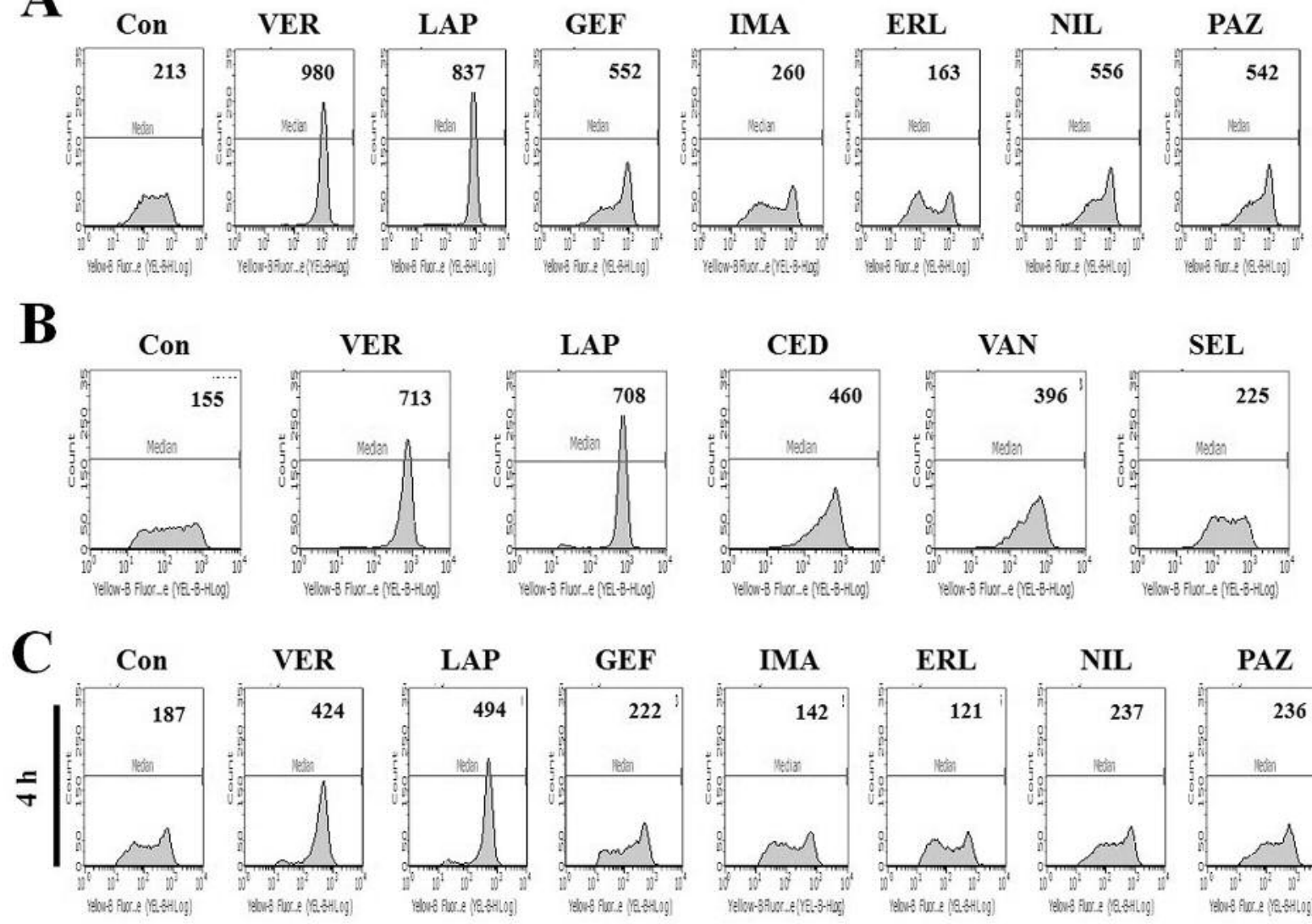

PAZ

Figure 3. Only lapatinib (LAP) has a high P-glycoprotein (P-gp)-inhibitory activity, whereas other tyrosine kinase inhibitors have low P-gp-inhibitory activity. A-C: KBV20C cells were grown on $60 \mathrm{~mm}$-diameter dishes and treated with $10 \mu M$ verapamil (VER), or $5 \mu M$ lapatinib, gefitinib (GEF), imatinib (IMA), erlotinib (ERL), nilotinib (NIL), pazopanib (PAZ), cediranib (CED), vandetanib (VAN), selumetinib (SEL), or 0.1\% dimethyl sulfoxide (DMSO; Con). After $4 \mathrm{~h}$ and $24 \mathrm{~h}$, all cells were stained with rhodamine and examined using fluorescence-activated cell sorting analysis, as described in the Materials and Methods. The value in each graph is the median of cellular distribution.

treatment of metastatic cancer (29-31). Previously, we found that the KBV20C cell line is a very useful model to study highly eribulin-resistant cancer (32).

As seen in Figure 2C, eribulin combined with $5 \mu \mathrm{M}$ of lapatinib, gefitinib, or erlotinib was similar to their effects when combined with vincristine treatments with regard to sensitizing effectiveness (Figure 1E). Eribulin-verapamil co-treatment at the same dose also had effects similar to those of vincristineverapamil co-treatment (Figures $1 \mathrm{E}$ and $2 \mathrm{C}$ ). These results demonstrate that treatment with lapatinib, gefitinib, or erlotinib is as effective in sensitizing resistant cancer cells to eribulin as well as vincristine. This finding also suggests that at low doses, the TKIs lapatinib, gefitinib, and erlotinib can be combined with other antimitotic drugs to sensitize P-gp-overexpressing cancer cells. We conclude that lapatinib, gefitinib, or erlotinib can be used in patients with various types of drug-resistant cancer.

Lapatinib has high P-gp-inhibitory activity, whereas other TKIs have low P-gp-inhibitory activity. In the next phase of our investigation, we evaluated the P-gp-inhibitory activity of TKIs in P-gp-overexpressing KBV20C cells since TKIs have been suggested for sensitizing of resistant cancer cells with high P-gp inhibitory activity $(15,16,18)$. We also expected that variation in the degree of P-gp inhibition among TKIs would be responsible for the difference in their sensitizing effects on VIC-treated KBV20C cells. We tested whether TKIs increased the inhibition of P-gp substrate efflux. Rhodamine 123, a well-known P-gp substrate, was used to measure P-gp inhibition (22-24). In this experiment, green fluorescence in the cell was indicative of intracellular accumulation of rhodamine 123 .

As shown in Figure 3A and B, lapatinib exhibited high P-gp-inhibitory activity, whereas other TKIs had much lower P-gp inhibitory activity. In a detailed quantitative analysis, the known P-gp inhibitor verapamil was used as a positive control $(5,7)$, and upon comparison we found that lapatinib at half the dose required for verapamil induced equivalent P-gp-inhibitory activity. This suggests that P-gp inhibition 

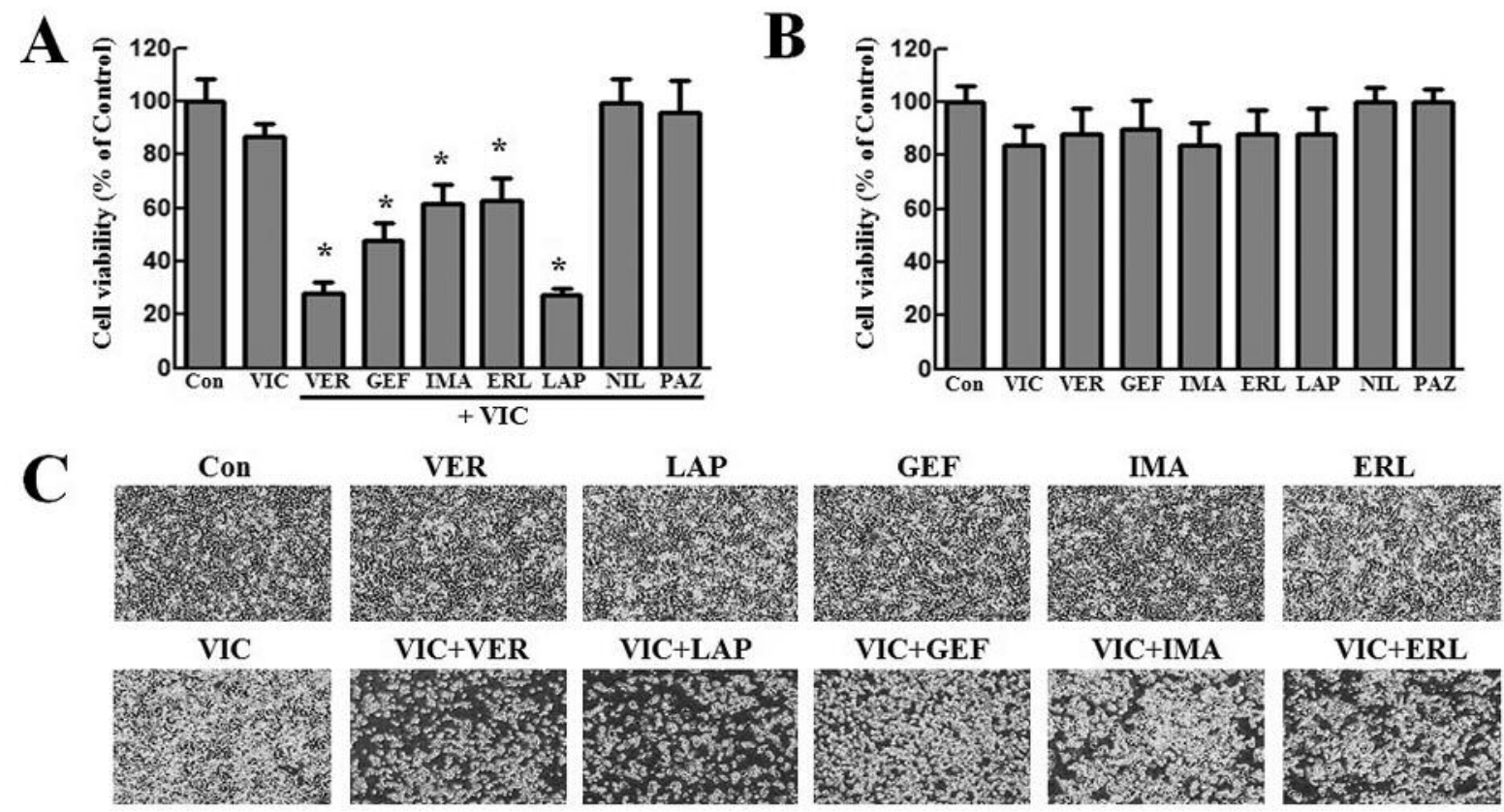

\section{VIC+IMA}

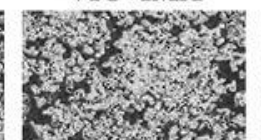

VIC+ERL
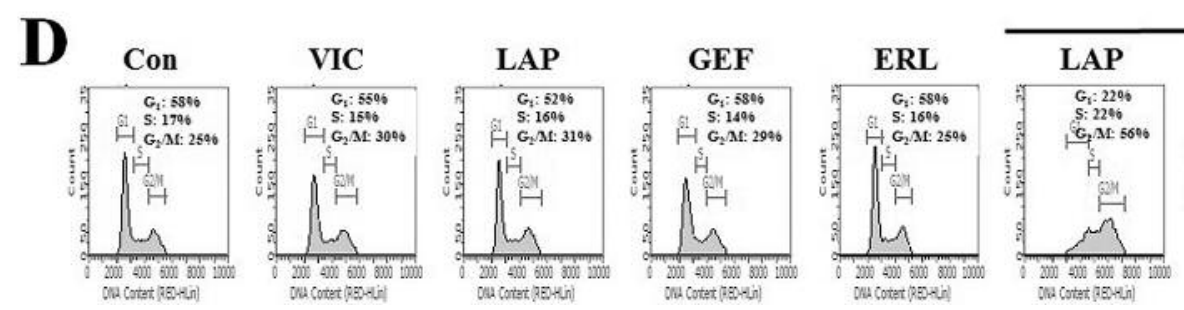

+ VIC

Figure 4. Lapatinib (LAP) at a low dose has a much greater vincristine (VIC)-sensitizing effect on KBV20C cells than other tyrosine kinase inhibitors. $A$ and B: KBV20C cells were plated on 96-well plates and grown to 30-40\% confluence. The cells were then stimulated for $48 \mathrm{~h}$ with $10 \mu \mathrm{M}$ verapamil (VER), or $2.5 \mu$ M lapatinib, gefitinib (GEF), imatinib (IMA), erlotinib (ERL), nilotinib (NIL), or pazopanib (PAZ) and in combination with $5 \mathrm{nM}$ vincristine, or $0.1 \%$ dimethyl sulfoxide (DMSO; Con). Cell viability assay was performed as described in the Materials and Methods. $C$ : KBV20C cells were grown on $60 \mathrm{~mm}$-diameter dishes and treated as described above. After 1 day, cells were examined using an inverted microscope at $\times 4$ magnification (scale bar $=100 \mu \mathrm{m}$ ). D: KBV20C cells were grown on $60 \mathrm{~mm}$-diameter dishes and treated with $2.5 \mu M$ lapatinib, gefitinib, or erlotinib alone and in combination with $5 \mathrm{nM}$ vincristine, or $0.1 \%$ DMSO (Con). After 24 h, fluorescence-activated cell sorting analyses were performed as described in Materials and Methods.

by lapatinib plays a key role in sensitizing cells to vincristine-lapatinib co-treatment. However, P-gp inhibition by other TKIs was much lower than that of verapamil or slightly higher than that of the control (Figure $3 \mathrm{~A}$ and $\mathrm{B})$. When we tested selumetinib, a serine/threonine kinase (19), we also found low P-gp-inhibitory activity (Figure 3B). As shown in Figure 3C, treatment with lapatinib or verapamil for $4 \mathrm{~h}$ led to results similar to those obtained after $24 \mathrm{~h}$ of treatment. This suggests that lapatinib inhibits P-gp by direct binding, similarly to the mechanism of verapamil. Considering that previously developed P-gp inhibitors, including verapamil, are toxic to normal cells $(5,7,8)$, we believe that lapatinib is a suitable alternative for sensitizing P-gp-overexpressing resistant cancer cells in clinical treatment.
The results also indicate that sensitizing KBV20C cells with low doses of vincristine-gefitinib is minimally affected by the P-gp-inhibitory effects of gefitinib. Notably, the level of P-gp inhibition by imatinib, and erlotinib was similar to that of the blank control (Figure 3A and C), suggesting that sensitizivity induced by vincristine-imatinib and vincristineerlotinib in KBV20C cells was independent of their P-gpinhibitory effects. Considering that even with very low or no P-gp-inhibitory activity, gefitinib, imatinib, or erlotinib still sensitized vincristine-treated KBV20C cells (Figure 3A and $\mathrm{C}$ ), they might be useful in clinical settings due to minimal toxic P-gp-inhibitory effects in normal cells.

Altogether, we found that these TKIs have different levels of P-gp inhibition and they function differently in sensitizing resistant KBV20C cells, which overexpress P-gp, to 
vincristine. Although co-treatments of vincristine with lapatinib, gefitinib, imatinib, and erlotinib had similarly high sensitizing effects on KBV20C cells, it is interesting that only lapatinib did so with very high P-gp-inhibitory activity, whereas the other TKIs induced sensitivity with little or no P-gp-inhibitory effect. Therefore, we conclude that these cotreatments have different mechanisms of sensitizing KBV20C cells.

At a low dose, lapatinib has much greater vincristinesensitizing effect on KBV20C cells than other TKIs. Our apoptosis results showed vincristine-lapatinib had a greater sensitizing effect than other co-treatments with TKIs (Figure 2B), suggesting that lapatinib is an ideal candidate in cotreatment for sensitizing resistant cancer cells. In order to demonstrate that lapatinib is better than other TKIs, we tested a lower dose $(2.5 \mu \mathrm{M})$ of TKIs for sensitizing KBV20C cells to vincristine. We believed that this lower dose along with VIC might better distinguish any variation in sensitizing effects among co-treatments with vincristine and TKIs.

As seen in Figure 4A, $2.5 \mu \mathrm{M}$ of lapatinib highly reduced viability in vincristine-treated KBV20C cells, whereas 2.5 $\mu \mathrm{M}$ of gefitinib, imatinib, or erlotinib led to about half that effect. Viability of cells treated with with vincristinelapatinib co-treatment was reduced by $>80 \%$ as compared to the control. When we compared the effects of lapatinib with the well-known P-gp inhibitor verapamil, $2.5 \mu \mathrm{M}$ lapatinib or $10 \mu \mathrm{M}$ verapamil had similar effectiveness when applied with vincristine to reduce KBV20C cell viability (Figure 4A). The results suggest that lapatinib is ideal, with a low half-maximal inhibitory concentration $\left(\mathrm{IC}_{50}\right)$, for sensitizing antimitotic drug-resistant cancer cells. There was no difference in viability between the control and treatment with individual TKIs (Figure 4B), suggesting that sensitizing by vincristine-lapatinib co-treatment resulted in synergistic effects in vincristine -resistant cancer cells.

We confirmed the results of these viability tests by microscopic observation. As shown in Figure 4C, $2.5 \mu \mathrm{M}$ of lapatinib was more effective than $2.5 \mu \mathrm{M}$ of gefitinib, imatinib, or erlotinib and had sensitizing effects similar to those of verapamil when combined with vincristine.

When cells in $\mathrm{G}_{2}$ arrest after treatment with low doses $(2.5 \mu \mathrm{M})$ of TKIs were quantitatively estimated (Figure 4D), we found that the proportion of $\mathrm{G}_{2}$-arrested cells comprised about $56 \%$ in cells treated with vincristine combined with lapatinib, $38 \%$ with gefitinib, and $33 \%$ with erlotinib. The results suggest that the effect of vincristine-lapatinib cotreatment in causing $G_{2}$ arrest was much greater than that of vincristine-gefitinib or vincristine-erlotinib.

Altogether, when we analyzed eight known TKIs to identify sensitizing of P-gp-overexpressing resistant cancer cells, we observed that lapatinib, gefitinib, imatinib, and erlotinib at low doses have greater sensitizing effects than the other drugs. Interestingly, gefitinib, imatinib, and erlotinib sensitized with little or no P-gp-inhibitory activity. In a detailed analysis with lower doses, we concluded that lapatinib, with high P-gp-inhibitory activity, may be a better and more potent agent in co-treatment for vincristine-treated resistant cancer cells than gefitinib, imatinib, or erlotinib. Therefore, lapatinib can be used to reduce drug toxicity and effectively sensitize cancer cells resistant to vincristine.

\section{Discussion}

Drug repositioning or repurposing is the application of known drugs for new indications. It has been used for the treatment of various diseases and has advantages, such as low cost and avoidance of many toxicity tests, which saves time (9-11). The urgent need for pharmacological treatments for resistant cancer can be efficiently addressed with drug repositioning and these drugs can be administered to patients sooner. In the current study, we investigated the novel application of some anticancer drugs (TKIs), repositioned for sensitizing P-gp-overexpressing resistant cancer cells.

Most importantly, we identified four TKIs, lapatinib, gefitinib, imatinib, and erlotinib, which can sensitize resistant KBV20C cells at a lower dose than other TKIs. Considering that half of the TKIs studied had vincristinesensitizing effects on resistant cancer cells at low doses, we conclude that TKIs generally have the ability to overcome resistance to antimitotic drugs. As EGFR or growth signaling receptor kinases are located in the cellular membrane (1214), we hypothesized that TKIs play a role in reducing or modifying increased P-gp activity in the membranes of resistant cancer cells. Although the resistant cancersensitizing ability of TKIs has been demonstrated (15-18), our research is pioneering in using selective TKIs as P-gp targeting drugs against cancer resistant to antimitotic drug. Our findings might also assist in selecting TKIs for preventing or reducing the occurrence of resistant cancer.

Our results were not limited to vincristine co-treatment because we confirmed that lapatinib, gefitinib, or erlotinib had sensitizing effects similar to those observed with eribulin in P-gp-overexpressing KBV20C cells. Eribulin was recently developed and is a promising drug for the treatment of resistant cancer (29-31). We previously reported that P-gpoverexpressing KBV20C cells are highly eribulin-resistant (32). Therefore, KBV20C cells are useful as models to study highly eribulin-resistant cancer. We found that that lapatinib, gefitinib, and erlotinib can sensitize KBV20C cells to eribulin-treated, suggesting that that lapatinib, gefitinib, and erlotinib can sensitize other antimitotic drug-resistant cancer cells similar to vincristine-treated KBV20C cells.

As the efflux of vincristine by P-gp is the main mechanism for resistance of KBV20C cells to vincristine, we hypothesized that the different vincristine-sensitizing effects of lapatinib, 
gefitinib, imatinib, and erlotinib co-treatment resulted from their P-gp-inhibitory effects. We demonstrated that lapatinib has a stronger P-gp-inhibitory effect than the well-known P-gp inhibitor verapamil, suggesting that vincristine-lapatinib sensitizing results from the inhibitory effects of lapatinib to prevent pumping out of vincristine. However, interestingly, we detected low levels of P-gp inhibition with gefitinib and no Pgp inhibition with imatinib and erlotinib, suggesting that gefitinib, imatinib, and erlotinib remove or inhibit factors that block vincristine effects in drug-resistant cancer cells and that their combination with vincristine thus has a synergistic effect on cells. Further investigation with gefitinib, imatinib, and erlotinib may be needed for determining the molecular targets for sensitizing resistant cancer cells without P-gp inhibition. As little or no P-gp-inhibitory activity was detected with gefitinib, imatinib, and erlotinib, an improved combination of chemotherapeutic agents can be developed for patients with cancer resistant to antimitotic drugs. As P-gp inhibitors have shown toxicity to normal cells $(5,7,8)$, we believe that gefitinib, imatinib, and erlotinib may be considered as combination drugs with little or no P-gp inhibition to sensitize the P-gp-overexpressing resistant cancer cells. As personalized medicines are currently gaining popularity, our findings with the TKIs lapatinib, gefitinib, imatinib, or erlotinib might contribute to effective prescriptions in patients with drug-resistant cancer who are allergic or sensitive to P-gp-inhibitory effects in normal tissues.

To conclude, our results highlight the novel selective sensitizing ability of TKIs. Furthermore, drug-resistant KBV20C cells that overexpress P-gp can be sensitized to the antimitotic drugs vincristine or eribulin by co-treatment with the cancer-targeting drugs lapatinib, gefitinib, imatinib, or erlotinib at low doses. Notably, gefitinib, imatinib, or erlotinib can sensitize the drug-resistant cancer cells with low P-gp-inhibitory activity. In addition, lapatinib, with a high P-gp inhibitory activity, is the best choice for increasing sensitizing of $\mathrm{P}$ gp-overexpressing KBV20C cells. As the toxicities of these drugs are already documented, they are readily available for use to treat patients with cancer. Our results could contribute to improvement in the efficacy of various chemotherapeutic agents used in combination for the treatment of patients with cancer which becomes resistant to chemotherapeutic drugs via $\mathrm{P}$-gp-overexpression.

\section{Conflicts of Interest}

The Authors declare no conflicts of interest regarding this study.

\section{Authors' Contributions}

Ji Yeong Kim: Collected the data, contributed data or analysis tools, wrote the article. Hyung Sik Kim: Contributed data or analysis tools. Sungpil Yoon: Conceived and designed the analysis, collected the data, contributed data or analysis tools, wrote the article.

\section{Acknowledgements}

This research was supported by National Research Foundation of Korea (NRF) funded by the Ministry of Education (NRF2017R1D1A1B03029158).

\section{References}

1 Jordan MA and Wilson L: Microtubules as a target for anticancer drugs. Nat Rev Cancer 4: 253-265, 2004. PMID: 15057285. DOI: $10.1038 / \mathrm{nrc} 1317$

2 Kim JH, Yoo HI, Kang HS, Ro J and Yoon S: Salinomycin sensitizes antimitotic drugs-treated cancer cells by increasing apoptosis via the prevention of G2 arrest. Biochem Biophys Res Commun 418: 98-103, 2012. PMID: 22244892. DOI: 10.1016/ j.bbrc.2011.12.141

3 McGrogan BT, Gilmartin B, Carney DN and McCann A: Taxanes, microtubules and chemoresistant breast cancer. Biochim Biophys Acta 1785: 96-132, 2008. PMID: 18068131. DOI: $10.1016 /$ j.bbcan.2007.10.004

4 Szakacs G, Paterson JK, Ludwig JA, Booth-Genthe C and Gottesman MM: Targeting multidrug resistance in cancer. Nat Rev Drug Discov 5: 219-234, 2006. PMID: 16518375. DOI: $10.1038 /$ nrd 1984

5 Chen Z, Shi T, Zhang L, Zhu P, Deng M, Huang C, Hu T, Jiang $\mathrm{L}$ and Li J: Mammalian drug efflux transporters of the ATPbinding cassette $(\mathrm{ABC})$ family in multidrug resistance: A review of the past decade. Cancer Lett 370: 153-164, 2016. PMID: 26499806. DOI: 10.1016/j.canlet.2015.10.010

6 Chufan EE, Kapoor K and Ambudkar SV: Drug-protein hydrogen bonds govern the inhibition of the ATP hydrolysis of the multidrug transporter P-glycoprotein. Biochem Pharmacol 101: 40-53, 2016. PMID: 26686578. DOI: 10.1016/ j.bcp.2015.12.007

7 Shukla S, Wu CP and Ambudkar SV: Development of inhibitors of ATP-binding cassette drug transporters: Present status and challenges. Expert Opin Drug Metab Toxicol 4: 205-223, 2008. PMID: 18248313. DOI: 10.1517/17425255.4.2.205

8 Yang $\mathrm{K}, \mathrm{Wu} \mathrm{J}$ and $\mathrm{Li} \mathrm{X}$ : Recent advances in the research of Pglycoprotein inhibitors. Biosci Trends 2: 137-146, 2008. PMID: 20103919.

9 Clark KB: New therapeutic bearings for repositioned drugs. Curr Top Med Chem 13: 2281-2282, 2013. PMID: 24059466.

10 Yoon S: A single treatment of selenate, a repositioning drug, specifically sensitizes P-gp-overexpressing resistant cancer cells. Cancer Cell Microenv 2(4): e957, 2015. DOI: 10.14800/ccm. 957

11 Pantziarka P and Cairns L: Recycling existing drugs for cancer therapy: delivering low cost cancer care. Ecancer Med Sci 8: ed40, 2014. PMID: 25075221. DOI: 10.3332/ecancer.2014.ed40

12. Roskoski R Jr.: The ErbB/HER family of protein-tyrosine kinases and cancer. Pharmacol Res 79: 34-74, 2014. PMID: 24269963. DOI: 10.1016/j.phrs.2013.11.002

13 Roskoski R, Jr.: A historical overview of protein kinases and their targeted small molecule inhibitors. Pharmacol Res 100: 123, 2015. PMID: 26207888. DOI: 10.1016/j.phrs.2015.07.010

14 Singh D, Attri BK, Gill RK and Bariwal J: Review on EGFR inhibitors: Critical updates. Mini Rev Med Chem 16: 1134-1166, 2016. PMID: 26996617. 
15 Beretta GL, Cassinelli G, Pennati M, Zuco V and Gatti L: Overcoming $\mathrm{ABC}$ transporter-mediated multidrug resistance: The dual role of tyrosine kinase inhibitors as multitargeting agents. Eur J Med Chem 142: 271-289, 2017. PMID: 28851502. DOI: $10.1016 /$ j.ejmech.2017.07.062

16 Wang YJ, Zhang YK, Kathawala RJ and Chen ZS: Repositioning of tyrosine kinase inhibitors as antagonists of ATP-binding cassette transporters in anticancer drug resistance. Cancers 6: 1925-1952, 2014. PMID: 25268163. DOI: 10.3390/ cancers 6041925

17 Collins DM, Crown J, O’Donovan N, Devery A, O'Sullivan F, O'Driscoll L, Clynes $\mathrm{M}$ and O'Connor R: Tyrosine kinase inhibitors potentiate the cytotoxicity of MDR-substrate anticancer agents independent of growth factor receptor status in lung cancer cell lines. Invest New Drugs 28: 433-444, 2010. PMID: 19499189. DOI: 10.1007/s10637-009-9266-0

18 Kuang YH, Shen T, Chen X, Sodani K, Hopper-Borge E, Tiwari AK, Lee JW, Fu LW and Chen ZS: Lapatinib and erlotinib are potent reversal agents for MRP7 (ABCC10)-mediated multidrug resistance. Biochem Pharmacol 79: 154-161, 2010. PMID: 19720054. DOI: 10.1016/j.bcp.2009.08.021

19 Kim JY, Welsh EA, Fang B, Bai Y, Kinose F, Eschrich SA, Koomen JM and Haura EB: Phosphoproteomics reveals MAPK inhibitors enhance MET- and EGFR-driven AKT signaling in KRAS-mutant lung cancer. Mol Cancer Res 14: 1019-1029, 2016. PMID: 27422710. DOI: 10.1158/1541-7786.MCR-150506

20 Cheon JH, Kim JY, Lee BM, Kim HS and Yoon S: P-gp Inhibition by XL019, a JAK2 inhibitor, increases apoptosis of vincristine-treated resistant KBV20C cells with increased p21 and pH2AX expression. Anticancer Res 37: 6761-6769, 2017. PMID: 29187454. DOI: 10.21873/anticanres.12136

21 Cheon JH, Kim KS, Yadav DK, Kim M, Kim HS and Yoon S: The JAK2 inhibitors CEP-33779 and NVP-BSK805 have high P-gp-inhibitory activity and sensitize drug-resistant cancer cells to vincristine. Biochem Biophys Res Commun 490: 1176-1182, 2017. PMID: 28669723. DOI: 10.1016/j.bbrc.2017.06.178

22 Kim JY, Park Y, Lee BM, Kim HS and Yoon S: P-gp Inhibition by the anti-psychotic drug pimozide increases apoptosis, as well as expression of $\mathrm{pRb}$ and $\mathrm{pH} 2 \mathrm{AX}$ in highly drug-resistant KBV20C cells. Anticancer Res 38: 5685-5692, 2018. PMID: 30275188. DOI: 10.21873 /anticanres.12905

23 Kim JY, Son JY, Lee BM, Kim HS and Yoon S: Aging-related repositioned drugs, donepezil and sildenafil citrate, increase apoptosis of anti-mitotic drug-resistant KBV20C cells through different molecular mechanisms. Anticancer Res 38: 5149-5157, 2018. PMID: 30194162. DOI: 10.21873/anticanres.12837

24 Kim JY, Tae IH, Lee BM, Kim HS and Yoon S: Low doses of the anti-psychotic drug aripiprazole have strong P-gp-inhibitory activity and sensitize anti-mitotic drug-resistant cancer cells Anticancer Res 38: 5101-5108, 2018. PMID: 30194155. DOI: 10.21873/anticanres. 12830
25 Park Y, Son JY, Lee BM, Kim HS and Yoon S: Highly eribulinresistant KBV20C oral cancer cells can be sensitized by cotreatment with the third-generation P-glycoprotein inhibitor, elacridar, at a low dose. Anticancer Res 37: 4139-4146, 2017. PMID: 28739698. DOI: 10.21873/anticanres.11801

26 Choi AR, Kim JH, Cheon JH, Kim HS and Yoon S: Attenuation of colchicine toxicity in drug-resistant cancer cells by cotreatment with anti-malarial drugs. Anticancer Res 36: 58595866, 2016. PMID: 27793909. DOI: 10.21873/anticanres.11171

27 Choi AR, Kim JH, Woo YH, Cheon JH, Kim HS and Yoon S: Co-treatment of LY294002 or MK-2206 with AZD5363 attenuates AZD5363-induced increase in the level of phosphorylated AKT. Anticancer Res 36: 5849-5858, 2016. PMID: 27793908. DOI: 10.21873/anticanres.11170

28 Lim JS, Park Y, Lee BM, Kim HS and Yoon S: Co-treatment with celecoxib or NS398 strongly sensitizes resistant cancer cells to antimitotic drugs independent of P-gp inhibition. Anticancer Res 36: 5063-5070, 2016. PMID: 27798865. DOI: 10.21873/ anticanres. 11075

29 Dell'Ova M, De Maio E, Guiu S, Roca L, Dalenc F, Durigova A, Pinguet F, Bekhtari K, Jacot W and Pouderoux S: Tumour biology, metastatic sites and taxanes sensitivity as determinants of eribulin mesylate efficacy in breast cancer: results from the ERIBEX retrospective, international, multicenter study. BMC Cancer 15: 659, 2015. PMID: 26449988. DOI: 10.1186/s12885015-1673-3

30 Dybdal-Hargreaves NF, Risinger AL and Mooberry SL: Eribulin mesylate: mechanism of action of a unique microtubule-targeting agent. Clin Cancer Res 21: 2445-2452, 2015. PMID: 25838395. DOI: $10.1158 / 1078-0432 . C C R-14-3252$

31 Inoue K, Saito T, Okubo K, Kimizuka K, Yamada H, Sakurai T, Ishizuna K, Hata S, Kai T and Kurosumi M: Phase II clinical study of eribulin monotherapy in Japanese patients with metastatic breast cancer who had well-defined taxane resistance. Breast Cancer Res Treat 157: 295-305, 2016. PMID: 27125669. DOI: $10.1007 / \mathrm{s} 10549-016-3808-\mathrm{x}$

32 Cheon JH, Lee BM, Kim HS and Yoon S: Highly halavenresistant KBV20C cancer cells can be sensitized by co-treatment with fluphenazine. Anticancer Res 36: 5867-5874, 2016. PMID: 27793910. DOI: 10.21873/anticanres.11172
Received April 29, 2019

Revised June 11, 2019

Accepted June 13, 2019 